\title{
Prediction for the first generation stars from a Galactic halo chemical evolution model with Miller-Scalo initial mass function
}

\author{
Y. $\mathrm{Lu}^{1,2}$, G. Zhao ${ }^{1}$, L. C. Deng ${ }^{1}$, M. R. Cen ${ }^{2}$, and Y. C. Liang ${ }^{1}$ \\ 1 Beijing Astronomical Observatory, Datun Rd. 20A, Chaoyang, Beijing 100012, PR China \\ 2 The Department of Physics, Normal University of Central China, Wuhan, Hubei 430079, PR China
}

Received 11 August 2000 / Accepted 18 December 2000

\begin{abstract}
A model of supernova-driven chemical evolution of the Galactic halo proposed by Tsujimoto et al. (1999) (TSY) is used to investigate the observations of the first generation stars (FGS). We assume in this paper that a star with a metallicity $[\mathrm{Fe} / \mathrm{H}] \leq-6$ is truly a FGS. We obtain FGS probabilities with the MillerScalo form of the stellar initial mass function and limit our model parameters to values consistent with the observed metallicity distributions of metal-poor stars in the range of $-4.0<[\mathrm{Fe} / \mathrm{H}]<-1.0$. We find a metallicity distribution function (MDF) of the long-lived halo field stars for $[\mathrm{Fe} / \mathrm{H}]<-1$ against the iron abundance in agreement with the observations. If the star formation in the halo is confined to individual gas clouds with masses from $110^{7}$ to $110^{8} M_{\odot}$, our model suggests that the probability of identifying FGS in the halo is about $6.1410^{-5}$ to $6.1410^{-6}$.
\end{abstract}

Key words. galaxy: evolution - galaxy: halo-stars: abundance - stars: formation - supernovae: general

\section{Introduction}

The first generation stars (FGS), i.e. the Population III (Pop III) stars, have strictly the chemical composition left by the Big Bang. Since FGSs are thought of as the fossil record of galaxy formation, even a completely unsuccessful search for Population III stars rewards astronomers with insight into a wide variety of astrophysical issues (Beers 1999). Up to a decade ago, searches for the FGSs had led to the result that the observational limit towards the lowest metallicities (Beers et al. 1985) is now about $[\mathrm{Fe} / \mathrm{H}]=-4$, i.e. 4 dex below the solar metallicity $Z_{\odot}=0.02$. One may wonder why, after those exhaustive survey of metal poor stars, only more than 100 stars with metallicities between $[\mathrm{Fe} / \mathrm{H}]=-4$ to $[\mathrm{Fe} / \mathrm{H}]=-3$ were found, while no Pop III stars, or even any stars with $[\mathrm{Fe} / \mathrm{H}]=-5$ were discovered. Many scenarios have been invoked to explain this (Truran \& Cameron 1971; Lin \& Murray 1992; De Marchi \& Paresce 1995a, 1995b; Cayrel 1986; Yoshii 1981; Yoshii et al. 1995).

In the standard big bang cosmology, the amount of heavy elements synthesized in the universe is as small as $Z \leq 10^{-10}$ ( $Z=$ mass fraction of heavy elements); hence the material from which the FGS were formed consists of pure hydrogen and helium (Wagoner et al. 1967). It is expected that the physical processes leading to the

Send offprint requests to: Y. Lu, e-mail: ly@yac.bao.ac.cn formation of the primordial stars are very different from those for Pop I stars formed from the gas with solar abundance. The initial mass function (IMF) of the primordial stars, for example, might be different from the Salpeter's IMF. However, the IMF of stars is key to modeling the evolution of galaxies; the IMF shape, together with the lower and upper bounds of stellar mass, largely influences the resulting abundance pattern of heavy elements ejected through supernova explosions, the total amount of mass contained in stellar remnants, the star formation rate and the chemical evolution models (Tinsley 1980).

The observational and theoretical state of FGS is not clear, leading to some confusion. In this work, we define a FGS as a star with a metallicity ratio of $[\mathrm{Fe} / \mathrm{H}] \leq-6$ (Beers 1999). A chemical evolution model allows us to theoretically analyse such metallicity distribution among generations of stars. Observational uncertainties and large number of parameters required by such models make their use eqivocal for very metal-poor stars (VMPS, i.e. halo stars). However, they can be used to determine a model for the evolving system using the most recent data and current hypotheses. In conventional chemical evolution models, the abundance pattern of stars is the same as the homogeneous gas cloud in which such stars are formed. The star formation rate (SFR) is assumed to be proportional to some power of the gas density (Tinsely 1980; Schmidt 1959). This has been successfully used to assess the 
chemical compositions of nearby stars and HII regions (Pagel \& Patchett 1975; Matteucci \& Greggio 1986; Yoshii et al. 1996).

Recent observations of VMPS in the Galactic halo imply that abundances pattern with $[\mathrm{Fe} / \mathrm{H}] \leq-2.5$ may indicate a preceding single or multiple supernova event (SNe) (McWilliam et al. 1995; Ryan et al. 1996). This suggests that conventional chemical evolution models cannot be applied to the Galactic halo. Thus, the abundance patterns of the VMPS or FGS is not expected to follow the predictions of the conventional models. Audouze \& Silk (1995) have attempted to explain the observed abundance patterns of VMPS. Shigeyama \& Tsujimoto (1998, ST98) and Tsujimoto \& Shigeyama (1998, TS98) proposed that the formation of VMPS is triggered by a single supernova remnant (SNR), and that the stars formed thus retain the abundance pattern of the SN. Following ST98 and TS98, Tsujimoto et al. (1999, hereafter TSY) developed a model for the early epochs of Galactic chemical evolution, suggesting that the metal-free Pop III stars could form in primordial gas clouds of the Galactic halo. If the stellar IMF exists in the form of Salpeter, we expect one FGS to be found per $10^{3}-10^{4}$ halo stars.

This paper aims to determine what stellar IMF properties are allowable which still fit the observational constraints using the TSY model. In Sect. 2, we discuss the characteristics of the Miller-Scalo IMF (Miller \& Scalo 1979). The chemical evolution model and assumptions are described in Sect. 3. In the last section we present the results and discussion.

\section{The initial mass function}

The IMF is assumed to be a time invariant mass spectrum with a power law of the form $n(m) \mathrm{d} m \propto m^{-(1+x)} \mathrm{d} m\left(m_{1} \leq\right.$ $m \leq m_{\mathrm{u}}$ ) (Salpeter 1955; Miller \& Scalo 1979; Tinsley 1980; Scalo 1986; Kroupa et al. 1993), where $n(m) \mathrm{d} m$ is the number of star in the mass interval $m$ to $m+\mathrm{d} m$, $m_{\mathrm{l}}$ is a lower mass limit of stars, and $m_{\mathrm{u}}$ is the upper mass limit of stars. Normally, the IMF is derived from the observed present-day mass function (PDMF) in the solar neighborhood, which is assumed to be independent of time (Scalo 1986). The derivation of the IMF from PDMF is difficult, involving assumptions about the star formation rate during the lifetime of the Galaxy (Tinsley 1980; Scalo 1986). For stars with lifetimes longer than the age of the Galaxy $\left(m \leq 1 M_{\odot}\right)$, the IMF is derived by assuming an average star formation rate in the past, whereas for stars with lifetimes negligible relative to the age of the Galaxy ( $m \geq 2 M_{\odot}$ ), the IMF is derived by assuming a presenttime star formation rate and taking into account the stellar lifetimes $(\tau(m))$. Given the uncertainties in both theory and observation, the IMF variations can be parameterized, and the proposed IMF can be tested by means of a detailed chemical evolution model. This method has been adopted in many cases (Yoneyama 1972; Hutchins 1976; Carlberg 1981; Kashlinsky \& Rees 1983; Palla et al. 1983; Silk 1983; Haiman et al. 1996; Uehara et al. 1996;
Tegmark et al. 1997; Omukai \& Nishi 1998; Nakamura \& Umemura 1999). Globular clusters (GCs) are expected to have a IMF similar to the Miller-Scalo form, with a typical lower mass limit of $0.1 M_{\odot}$ (Padoan et al. 1997, PNJ). It is worth noting that halo field stars, associated with type II supernovae, indicate an origin common to that of GCs (Wheeler et al. 1989). Based on the chemical evolution model of TSY, we use the Miller \& Scalo IMF to model observations of Galactic halo stars. This IMF can be well approximated by a half-Gaussian distribution in $\log m$, which is,

$\phi(\log m)=C_{0} \exp \left[-C_{1}\left(\log m-C_{2}\right)^{2}\right]$,

where $C_{0}=66.2, C_{1}=1.15, C_{2}=-0.88 . \mathrm{m}$ is the mass of a MS star in units of $M_{\odot}$.

\section{A chemical evolution model of the Galactic halo}

\subsection{Basic equations and assumptions}

We assume that the star-forming process is confined in isolated clouds, which may explain the recent observation of abundance patterns in metal-poor stars (TS98; ST98; TSY). In our model, a fraction, $x_{\mathrm{III}}$, of the cloud mass has been turned into Pop III stars at time $t=0$; the massive stars of the same population have exploded as Pop III SNs, which in turn initiate the chemical evolution in a given cloud. All stars of subsequent generations are assumed to form from the SNR shells around the radiative shock front. The mass fraction of each shell that is converted into stars is taken as a constant, $\varepsilon$. Following TSY, when stars form as above, the star formation rate (SFR) $\dot{M}_{*}(t)$ can be given by

$$
\begin{aligned}
\dot{M}_{*}(t)= & {\left[\frac{\phi\left(m_{\mathrm{t}}\right)}{m_{\mathrm{t}}}\right]\left|\frac{\mathrm{d} m_{\mathrm{t}}}{\mathrm{d} t}\right| x_{\mathrm{III}} M_{\mathrm{c}} \quad \text { for } \quad t \leq \tau(m), } \\
\dot{M}_{*}(t)= & \int_{\max \left(m_{\mathrm{t}}, m_{\mathrm{SN}, 1}\right)}^{m_{\mathrm{u}}} \mathrm{d} m \varepsilon M_{\mathrm{sh}}(m, t) \frac{\phi(m)}{m} \\
& \times \dot{M}_{*}[t-\tau(m)] \text { for } t>\tau(m),
\end{aligned}
$$

where $\tau(m)$ denotes the lifetime of a star with mass $m$, $m_{\mathrm{t}}$ is the stellar mass for which $\tau(m)=t$. The masslifetime relation is $\log \left(m_{\mathrm{t}} / M_{\odot}\right)=0.0558[\log (t / \mathrm{yr})]^{2}-$ $1.338 \log (t / \mathrm{yr})+7.764$ (Renzini \& Buzzoni 1986). The Miller-Scalo IMF $\phi(m)$ is adopted with the upper and lower mass limits of $m_{\mathrm{u}}=50 \mathrm{M}_{\odot}$ and $m_{\mathrm{l}}=0.1 \mathrm{M}_{\odot}$. A lower mass limit of stars that explode as $\mathrm{SN}$ is taken to be $m_{\mathrm{SN}, 1}=10 M_{\odot}$. The mass of the shell is given by $M_{\mathrm{sh}}(m, t)=M_{\mathrm{ej}}(m)+M_{\mathrm{sw}}(m, t)$, where $M_{\mathrm{ej}}(m)$ is the mass of the SN ejecta and $M_{\mathrm{sw}}(m, t)$ is the mass of the gas swept up by the SNR. Since $M_{\mathrm{sw}}(m, t)$ is insensitive to $m$ and $t$, we adopt here $M_{\mathrm{Sw}}(m, t)=6.510^{4} M_{\odot}$ (ST98, TSY). The formulae of the mass $M_{\mathrm{g}}(t)$ and the metallicity $Z_{\mathrm{g}}(t)$ of the gas changing with time is the same as 
Eqs. (2) and (3) of TSY, respectively. For the initial conditions of our model, we assume a halo consisting of gas and Pop III stars with primordial abundance. We adopt a constant time-step of $310^{6}$ years, which is longer than the dynamical evolution timescale of a SNR and shorter than the lifetime of the most massive stars. The number of stars formed in each time-step includes stars formed in all the shells. Newly born stars inherit the abundance pattern of the interstellar medium (ISM) out of which they are formed, therefore carrying information of the ISM at the place and time of their birth. Stars in the range of $10 M_{\odot}$ to $50 M_{\odot}$ will explode as SN II, resulting in an enrichment of the neighboring ISM. Stellar yields are taken from Woosley \& Weave (1995), which we interpolate linearly for the finer mass-grid in our simulation. For SN with masses below $13 M_{\odot}$, stellar yields are not available. Since the nucleosynthesis models show declining yields towards lower mass progenitors, the stellar yields of a $10 M_{\odot}$ is set to $1 / 1000$ of that of a $13 M_{\odot}$ and then interpolated linearly for stellar masses in between. (Argast et al. 2000). We do not consider SN Ia, since we are only interested in the very early enrichment of the halo ISM, which is dominated by SN II. Intermediate mass stars will evolve to planetary nebulae, returning only slightly enriched material in the course of their evolution, and will not change the element abundance significantly. Low mass stars do not evolve significantly during the time scale under consideration (Argast et al. 2000). Thus, we ignore the influences of the intermediate mass stars and low mass stars on the enrichment pattern of the ISM.

\section{Results and discussions}

\subsection{Results}

From the model presented in the preceding section, we calculate the chemical evolution of the Galactic halo. The two free parameters in our model are: 1 ) the mass fraction $x_{\text {III }}$ of metal-free Pop III stars initially formed in each cloud; and 2) the mass fraction $\varepsilon$ of stars formed in a dense shell of each SNR. We choose the values of these parameters in order to reproduce the observed $[\mathrm{Fe} / \mathrm{H}]$ distribution function of halo stars for $[\mathrm{Fe} / \mathrm{H}]<-1.0$, tuned as per TSY. We found that $x_{\text {III }}$ must be less than about $110^{-5}$. A value of $\varepsilon=9.7510^{-4}$ gives the best fit to the observed $[\mathrm{Fe} / \mathrm{H}]$ distribution function for various values of $x_{\mathrm{III}}\left(<10^{-5}\right)$. The discovery probability $p_{\text {III }}$ of the Pop III stars (defined as a fraction of the number of Pop III stars among all the long-lived stars ever formed with $\left.m<1 M_{\odot}\right)$ decreases with increasing duration $\Delta T$ of star formation in a cloud (see Fig. 1). Each value of $p_{\text {III }}$ corresponds to about 10 times $x_{\text {III }}$, because about $10 \%$ of the fractional mass of gas $M_{\mathrm{g}}(0)$ becomes converted into stars At lease one massive Pop III star must explode in the initial phase for SN-induces star formation to proceed. Therefore, for each mass $M_{\mathrm{c}}$ of a cloud, a lower limit of $p_{\text {III }}$ exists. The strict lower limit of $p_{\mathrm{III}}$ for each $M_{\mathrm{c}}$ of a cloud is listed in Table $1 . M_{\mathrm{c}}$ is in the range of several $10^{5}-10^{8} M_{\odot}$,

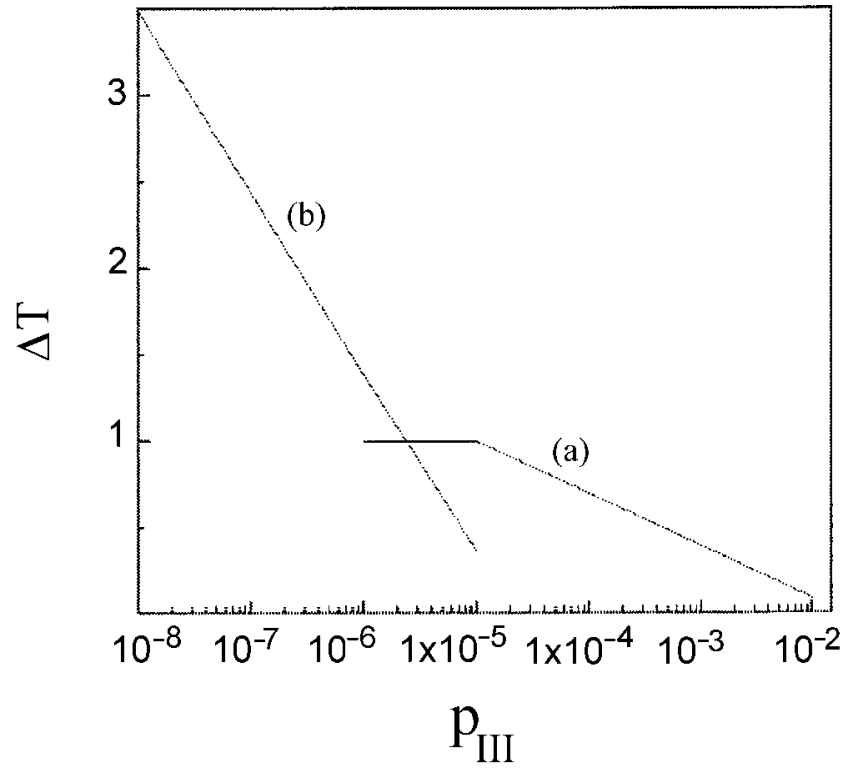

Fig. 1. The relation between the duration $\Delta T$ of star formation in a cloud and a probability $p_{\text {III }}$ of observing Pop III stars as a fraction of their number among all the long-lived stars with $m<1 M_{\odot}$. a) stands for Salpeter's IMF, and b) is for MillerScalo's IMF

Table 1. The relation between the strict lower bound of $p_{\text {III }}$ and each mass $M_{\mathrm{c}}$ of a cloud, the ratio of $p_{\text {III }}$ with $x_{\text {III }}$

\begin{tabular}{|c|c|c|}
\hline$M_{\mathrm{c}}$ & $p_{\text {III }}$ of present model & $p_{\text {III }}$ of TSY model \\
\hline $110^{5} M_{\odot}$ & $6.1410^{-3}$ & $2.5310^{-2}$ \\
\hline $110^{6} M_{\odot}$ & $6.1410^{-4}$ & $2.5310^{-3}$ \\
\hline $110^{7} M_{\odot}$ & $6.1410^{-5}$ & $2.5310^{-4}$ \\
\hline $110^{8} M_{\odot}$ & $6.1410^{-6}$ & $2.5310^{-5}$ \\
\hline
\end{tabular}

as inferred from masses of the Galactic globular clusters. Using this cloud mass, the lower bound of the Pop III star dicovery probability is $6.1410^{-3}$ to $6.1410^{-6}$.

Figure 2 shows the predicted stellar $[\mathrm{Fe} / \mathrm{H}]$ distribution function for given $x_{\mathrm{III}}$, compared to the data obtained by Ryan \& Norris (1991). The solid circle is the present model with $x_{\mathrm{III}}=10^{-5}$ and $M_{\mathrm{c}}=310^{8} M_{\odot}$. The solid triangle is the TSY model with $x_{\text {III }}=10^{-5}$ and $M_{\mathrm{c}}=310^{8} M_{\odot}$. The star is the observational data (Ryan \& Norris 1991). With decreasing heavy-element yield, such as mass loss from the halo (Hartwick 1976; Bond 1981; Laird et al. 1988; Ryan $\&$ Norris 1991), we obtain an agreement with the data for $[\mathrm{Fe} / \mathrm{H}]<-1$ using the Miller-Scalo IMF. Star formation terminates at the gas metallicity of $[\mathrm{Fe} / \mathrm{H}] \sim-1.5$, reproducing the observed stellar frequency $[\mathrm{Fe} / \mathrm{H}] \sim-1.53$. We show the mass fraction of the initial gas varied with time in Fig. 3. In Fig. 3, (a) is the TSY model and (b) is our present model. Figure 4 shows the predicted $[\mathrm{Fe} / \mathrm{H}]$ distribution function of stars formed at a given age in the age- $[\mathrm{Fe} / \mathrm{H}]$ plane. In Figure 4, the solid line (a) corresponds to the TSY model, and the solid line (b) to our present model. 


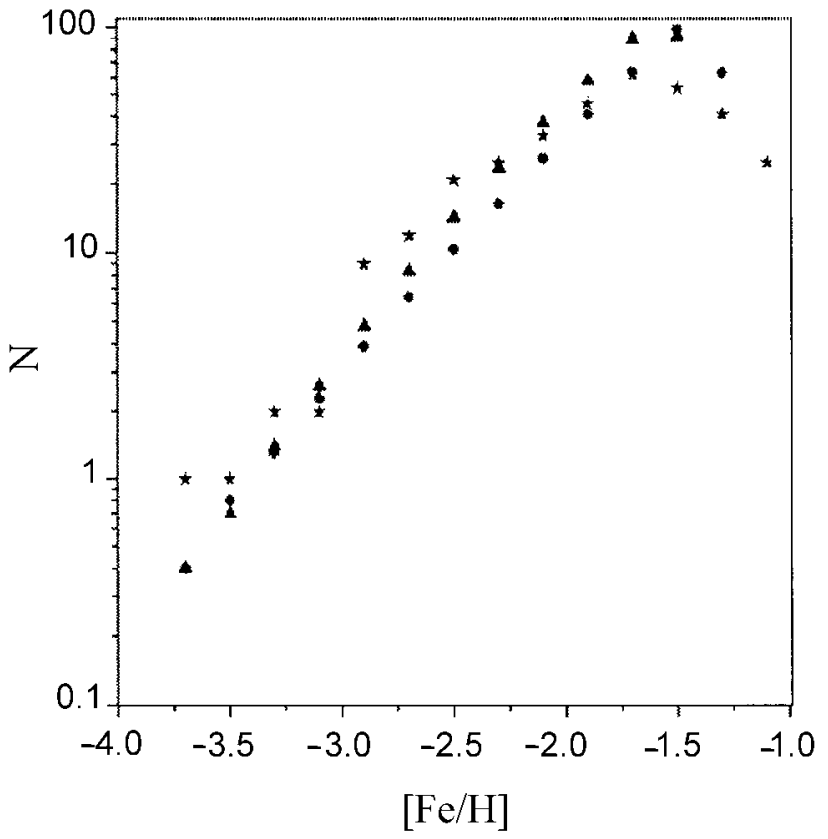

Fig. 2. The frequency distribution of the long-lived halo field stars for $[\mathrm{Fe} / \mathrm{H}]<-1$ against the iron abundance, compared with the observations of Ryan \& Norris (1991). The star corresponds to the observational data. The solid circle is a calculation from the present model, and the solid triangle corresponds to the model of TSY

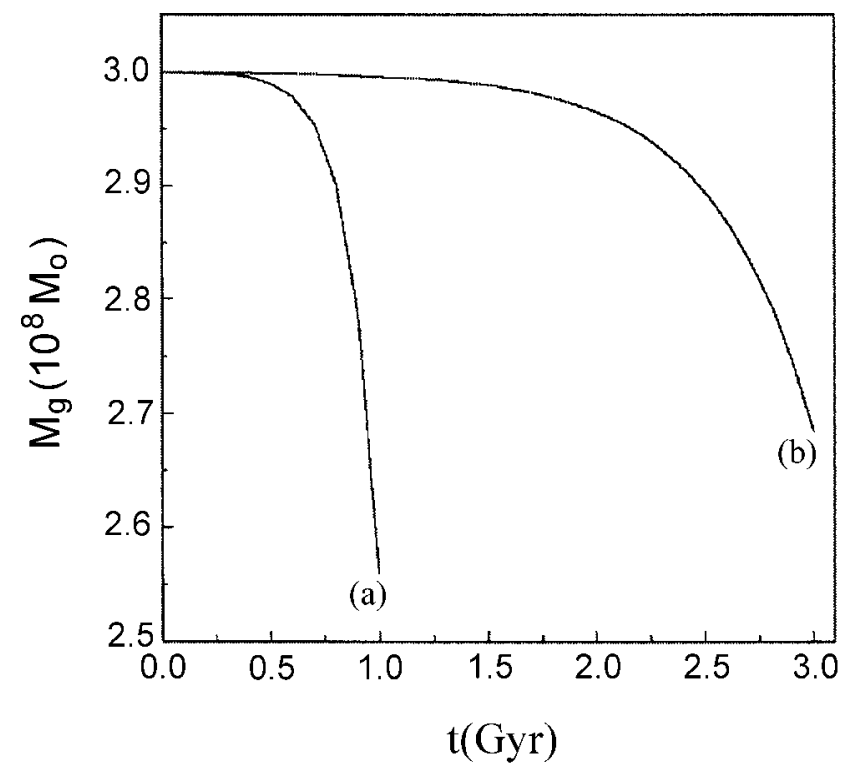

Fig. 3. The mass fraction of the gas evolves with time. a) The TSY model, b) the present model

\subsection{Discussion}

Based on a new model for Galactic chemical evolution (TSY), but using the Miller-Scalo form of the primordial IMF, our results are similar to those of the TSY model. However, the probability of detecting the metal-free Pop III stars is smaller because the Miller-Scalo IMF is enhanced in the massive end compared with the

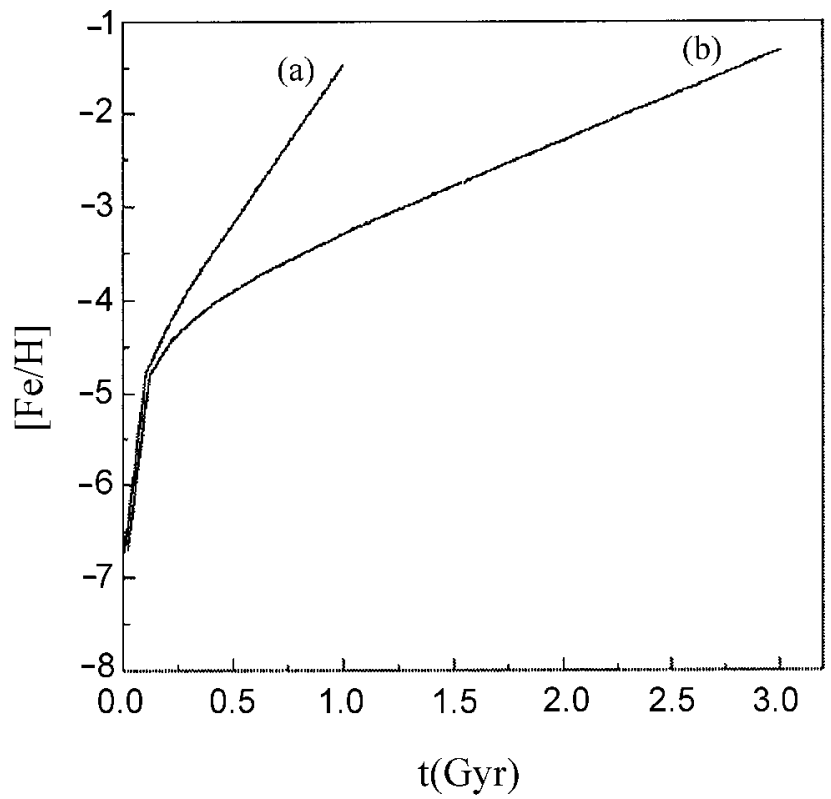

Fig. 4. The model curves of the metallicity of ISM over time. The solid line a) corresponds to the TSY model, and the solid line $\mathbf{b}$ ) is the present model curve

Salpeter IMF at time $t=0$. This can be infered from the longer star-formation duration $\Delta T$ (Fig. 1) and the smaller fraction $x_{\text {III }}$ of the cloud mass turned into Pop III stars at time $t=0$ in the frame of Miller-Scalo IMF. From Table 1, we find that the probability of detecting the metal-free Pop III stars with the Miller-Scallo IMF is about 0.24 times that found with the Salpeter IMF. In another words, we find only one Pop III star in a sample of $410^{4}$ halo stars here.

In order to reproduce the observed $[\mathrm{Fe} / \mathrm{H}]$ distribution function of stars, we also need to decrease the mean value of heavy-element yields (about -0.35 dex) from SN. This is different from the TSY model. Usually, theoretical metallicity distributions of stars are constructed as a function of metallicity $[Z / H]=\log \left(n_{Z} / n_{H}\right)_{*}-\log \left(n_{Z} / n_{H}\right)_{\odot}$, whereas observations of stellar abundances are usually expressed in terms of $[\mathrm{Fe} / \mathrm{H}]$, since the abundance of iron is the most easily measured. For halo stars, $[\mathrm{Fe} / \mathrm{H}] \neq[Z / H]$, because not all elements are deficient by the same factor. The chemical evolution models adopted in our paper is parameterized by the "effective yield", which is a measure of the efficiency of the enrichment process. The yield determined from observed $[\mathrm{Fe} / \mathrm{H}]$ values is not the effective yield of metals, but rather that of iron. Following the argument of Lambert (1989) and Kurucz (1979), we also assumed that the effective yield of metals will be 0.35 dex higher, so that $[\mathrm{Fe} / \mathrm{H}]$ is $[\mathrm{Fe} / \mathrm{H}]=[Z / H]-0.35$.

If the form of the primordial IMF is the Miller-Scalo form, finding the very first generation of star is difficult as found above. Note that the definition of FGS implies a very specific set of requirements for pollution of stellar atmospheres (either by intrinsic or extrinsic mechanisms) and the IMF: (i) Pollution of a stellar atmosphere with 
metallic species subsequent to a star's formation cannot be effective (see the confined values of $\varepsilon$ ), else we would never measure a star's atmospheric metal abundance satisfying the definition of FGS; (ii) The IMF must extend to include low stars with sufficiently long main-sequence lifetimes that might be identifiable at present. In the case of top-heavy stellar IMF in the galaxy at early times, no metal-free stars can be found. In conclusion, metal-free Pop III stars are sure to be found eventually if the star formation history in the Galactic Halo is the same as that assumed by TSY, and by increasing the sample of observed stars with the lowest metal abundances.

Acknowledgements. We thank R. Cayrel for his important comments and $\mathrm{T}$. Tsujimoto for a useful discussion and friendly help to our paper. This work is partially supported by the National Natural Science Foundation of China.

\section{References}

Argast, D., Samland, M., Gerhard, O. E., \& Thielemann, F. K. 2000, A\&A, 356, 873

Audouze, J., \& Silk, J. 1995, ApJ, 451, L49

Beers, T. C. 2000, AAS, 196, 3509B

Beers, T. C., Preston, G. W., \& Shectman, S. A. 1992, AJ, 103, 1987 (BPS)

Carlberg, R. G. 1981, MNRAS, 197, 1021

Cayrel, R. 1986, A\&A, 168, 81

De Marchi, G., \& Paresce, F. 1995a, A\&A, 304, 202

De Marchi, G., \& Paresce, F. 1995b, A\&A, 304, 211

Haiman, Z., \& Loeb, A. 1997, ApJ, 483, 21

Hutchins, J. B. 1976, ApJ, 205, 103

Kashlinsky, A., \& Rees, M. J. 1983, MNRAS, 205, 955

Kroupa, P., Tout, C. A., \& Gilmore, G. 1993, MNRAS, 262, 545

Kurucz, R. L. 1979, ApJS, 40, 1
Lambert, D. L. 1989, in Cosmic Abundances of Matter, ed. C. J. Waddington (AIP, New York), 168

Lin, D. N. C., \& Murray, S. D. 1992, ApJ, 394, 523

Matteucci, F., \& Greggio, L. 1986, A\&A, 154, 279

McWilliam, A., Preston, G. W., Sneden, C., \& Searle, L. 1995, AJ, 109, 2757

Miller, G. E. \& Scalo, J. M. 1979, ApJS, 41, 513

Nakamura, F., \& Umemura, M. 1999, ApJ, 515, 239

Omukai, K., \& Nishi, R. 1998, ApJ, 508, 141

Pagel, B. E., \& Patchett, B. E. 1975, MNRAS, 172, 13

Padoan, P., Nordlund, A. A., \& Jones, B. J. T. 1997, MNRAS, 288, 145 (PNJ)

Palla, F., Salpeter, E. E., \& Stahler, S. W. 1983, ApJ, 271, 632

Renzini, A., \& Buzzoni, A. 1986, Seg. Work, 195

Ryan, S. G., \& Norris, J. E. 1991, ApJ, 101, 5

Ryan, S. G., Norris, J. E., \& Beers, T. C. 1996, ApJ, 471, 254

Salpeter, E. E. 1955, ApJ, 121, 161

Scalo, J. M. 1986, Fund. Cosmic Phys., 11, 1

Schmidt, M. 1959, ApJ, 129, 243

Shigeyyama, T., \& Tsujimoto, T. 1998, ApJ, 507, L135 (ST98)

Silk, J., 1983, MNRAS, 205, 705

Tegmark, M., Silk, J., Rees, M. J., Blanchard, A., Abel, T., \& Palla, F. 1997, ApJ, 474, 1

Tinsley, B. M. 1980, Fund. Cosmic Phys., 5, 287

Truran, J. W., \& Cameron, A. G. W. 1971, APSS, 14, 179

Tsujimoto, T., \& Shigeyama, T. 1998, ApJ, L151 (TS98)

Tsujimoto, T., Shigeyama, T., \& Yoshii, Y. 1999, ApJ, 519, L63 (TSY)

Uehara, H., Susa, H., Nishi, R., Yamada, M., \& Nakamura, T. 1996, ApJ, 473, L95

Wagoner, R. V., Fowler, F., \& Hoyle 1976, ApJ, 148, 3

Wheeler, J. C., Sneden, C., James, W., \& Truran, Jr. 1989, ARA\&A, 27, 279

Woosley, S. E., \& Weaver, T. A. 1995, ApJS, 101, 181

Yoneyama, T. 1972, PASJ, 24, 87

Yoshii, Y. 1981, A\&A, 97, 280

Yoshii, Y., Mathews, G. J., \& Kajino, T. 1995, ApJ, 447, 184

Yoshii, Y., Tsujimoto, T., \& Nomoto, K. 1996, ApJ, 462, 266 